\title{
Current status and potential application of navigated transcranial magnetic stimulation in neurosurgery: a literature review
}

\author{
Xiaojing Fang ${ }^{1}$, Meige Liư ${ }^{4}$ Changyu Lư ${ }^{3}$ Y Yuanli Zhao ${ }^{2,3}$ and Xianzeng Liu*
}

\begin{abstract}
Transcranial magnetic stimulation (TMS) is a noninvasive neurophysiologic technique that can stimulate the human brain. Positioning of the coil was often performed based merely on external landmarks on the head, meaning that the anatomical target in the cortex remains inaccurate. Navigated transcranial magnetic stimulation (nTMS) combines a frameless stereotactic navigational system and TMS coil and can provide a highly accurate delivery of TMS pulses with the guidance of imaging. Therefore, many novel utilities for TMS could be explored due to the ability of precise localization. Many studies have been published, which indicate nTMS enables presurgical functional mapping. This review aimed to provide a comprehensive literature review on nTMS, especially the principles and clinical applications of nTMS. All articles in PubMed with keywords of "motor mapping," "presurgical mapping," "navigated transcranial magnetic stimulation," and "language mapping" published from 2000 to 2018 were included in the study. Frequently cited publications before 2000 were also included. The most valuable published original and review articles related to our objective were selected. Motor mapping of nTMS is validated to be a trustful tool to recognize functional areas belonging to both normal and lesioned primary motor cortex. It can offer reliable mapping of speech and motor regions at cortex prior to operation and has comparable accuracy as direct electrical cortical stimulation. nTMS is a powerful tool for mapping of motor and linguistic function prior to operation, has high application value in neurosurgery and the treatment of neurological and psychiatric diseases, and has gained increasing acceptance in neurosurgical centers across the world.
\end{abstract}

Keywords: Transcranial magnetic stimulation, Motor mapping, Speech mapping, Neurosurgery

\section{Main text}

Since the introduction for stimulating the human motor cortex by transcranial magnetic stimulation (TMS) in 1985 [1], TMS has been applied in studying the processing of cortex information and treating psychiatric and neurologic diseases [2-6]. However, how to precisely locate the magnetic coil at cortical regions remains a major obstacle in relevant investigations. In most cases, the anatomic features of involved subjects were not taken into consideration in TMS studies, so it is very difficult to reach the intended area accurately [7].

In the past decades, a combination of optically tracked stereotactic navigation systems and TMS technology was

\footnotetext{
* Correspondence: liuxianzeng@pkuih.edu.cn

'Department of Neurology, Peking University International Hospital, 1 Life

Science St, Changping District, Beijing 102206, China

Full list of author information is available at the end of the article
}

developed. This system can form a picture of the stimulation sites through the three dimensions (3D) rebuilt MRI data of the subjects' brain, which was therefore named as navigated transcranial magnetic stimulation (nTMS) $[8,9]$. NTMS has been tested for mapping of the motor cortex prior to operation and is widely accepted by neurosurgeons [10-12]. Motor mapping by nTMS correlate well with mapping of direct electrical stimulation (DES) during surgery, especially when compared to other preoperative mapping methods such as functional magnetic resonance imaging (fMRI) or magnetoencephalography $[13,14]$. In recent years, nTMS has been widely used in motor mapping, and more evidence shows that it is beneficial for patients with brain tumor. Many studies observed better outcomes and higher survival rates in brain tumor patients who use

(c) The Author(s). 2019 Open Access This article is distributed under the terms of the Creative Commons Attribution 4.0 International License (http://creativecommons.org/licenses/by/4.0/), which permits unrestricted use, distribution, and reproduction in any medium, provided you give appropriate credit to the original author(s) and the source, provide a link to the Creative Commons license, and indicate if changes were made. The Creative Commons Public Domain Dedication waiver (http://creativecommons.org/publicdomain/zero/1.0/) applies to the data made available in this article, unless otherwise stated. 
nTMS before surgery, thus expanding the initial role of nTMS as a mere preoperative planning tool $[15,16]$.

We review the basic principles and clinical applications of nTMS, protocols for motor and language mapping, comparison between nTMS and other preoperative mapping modalities, and future applications of nTMS, so as to improve the understanding and clinical application of nTMS.

\section{Electric-field navigation and line navigation}

Many techniques can be applied to the navigation of TMS. They can be divided into two groups, namely, line-navigated transcranial magnetic stimulation (Ln-TMS) and electric-field navigated transcranial magnetic stimulation (En-TMS). Ln-TMS exhibits the location of the coil while not showing coil angulation in relation to the patient's head. The stimulation spot is assumed to be on the line that passes through the geometric center of the coil and is perpendicular to the coil surface [17], and if the coil is not perfectly tangential with respect to the skull, the target would be imprecise [18]. En-TMS can calculate the electric field on the cortex while stimulating. It can visualize and calculate the electric field with its dose and orientation, as well as optimize the coil positioning continuously. The resolution of En-TMS reaches $2 \mathrm{~mm}[11,16]$. At present, only the En-TMS system could be used for tumor-patient diagnostics [11-13, 19-21]. As what were already clarified for En-TMS, more investigations using intraoperative DES are needed to determine whether Ln-TMS can produce motor mapping with high specificity and accuracy in neurosurgical operations.

\section{Motor mapping and speech mapping protocols}

Preparation for the session:

Upload individual MRI data and use them to generate a 3D head model. Match the 3D head model with the patients' head (Fig. 1).

Hot spot identification:

The rapid changes of transcranial magnetic field produce electric current at a specific region at the cortex, leading to a corresponding motor evoked potential (MEP) [22]. Hot spot identification is the search for the cortical sites producing maximum MEPs [23, 24].

RMT (resting motor threshold) determination:

RMT refers to the lowest intensity of TMS which is able to elicit a $50-\mu \mathrm{V}$ MEP in a relaxed small hand muscle in 5 out of 10 stimulations. The target muscle should be at rest, and monitoring of muscle activity is critical during the determination of the RMT [25].

Set the stimulate mode to single pulse TMS and set the output of stimulator to $110 \%$ RMT when doing motor mapping. Stimulate the central sulcus, precentral sulcus, postcentral sulcus, and precentral gyrus with a 2-3-mm interval [26]. Mark the nTMS positive spots on the cortex (Fig. 1).

Most language mapping protocols use object naming and navigated repetitive TMS (nrTMS) combined with video recording of the behavioral results. The stimulate strength is $100-120 \%$ of the RMT for the hand muscles. The stimulate spot is not restrained to conventional regions stated by the Geschwind's language model [27, 28] The corresponding nrTMS locations in the parcellated cortex are marked as language-related and are tagged by the observed error type $[29,30]$.

\section{Safety issue}

It has been proved that nTMS is safe in both normal subjects and patients with intracranial lesions. The only absolute contraindication of nTMS is the presence of metal or electronic devices, such as cochlear implants, pulse generators, and medical pumps, near the coil stimulation site, which are at risk of being destroyed [31]. A cohort study involving 733 patients showed that nTMS was well tolerated in motor mapping, with only $6 \%$ of patients feeling unwell. In language mapping, as nrTMS was used, 93\% of the patients felt uncomfortable and $70 \%$ of the patients felt pain. The discomfort of repetitive stimulation was mainly caused by contraction of the temporalis muscle and orbicularis oculi muscle and direct stimulation of trigeminal nerve sensory branches $[32,33]$. There are few reports on seizure induced by single-pulse TMS in patients with neurological diseases; therefore, it should be noted that patients with cerebral lesions might still have a high risk of seizure induced by TMS [34].

\section{Comparison between nTMS and direct electrical cortical stimulation}

DES has always been accepted as the gold standard for motor mapping and language mapping [35]. It was reported that motor activation points detected by nTMS were closely associated with those found in DES [36, 37]. One meta-analysis reviewed six studies with a total of 81 patients and summarized that the mean distance of motor cortex identified on DES and nTMS was 6.18 $\mathrm{mm}$ [37]. Two other studies mapped the motor cortex in patients with growing malignant and large tumors, and they found that the distances of DES and nTMS were $10.5 \mathrm{~mm}$ and $7.83 \mathrm{~mm}$, respectively [11, 12]. Pavia reported a distance of approximately $4 \mathrm{~mm}$ in a group of patients with low-grade gliomas. This may be explained by deficiency of accompanied edema and the relative small size of the lesions [38].

Language mapping conducted by NrTMS has shown close association with DES, and compared with DES, nTMS has a sensitivity value of $90 \%$, specificity of $98 \%$, negative predictive value of $99 \%$, and positive predictive value of $69 \%$ [31, 39-41]. NrTMS can be completed 


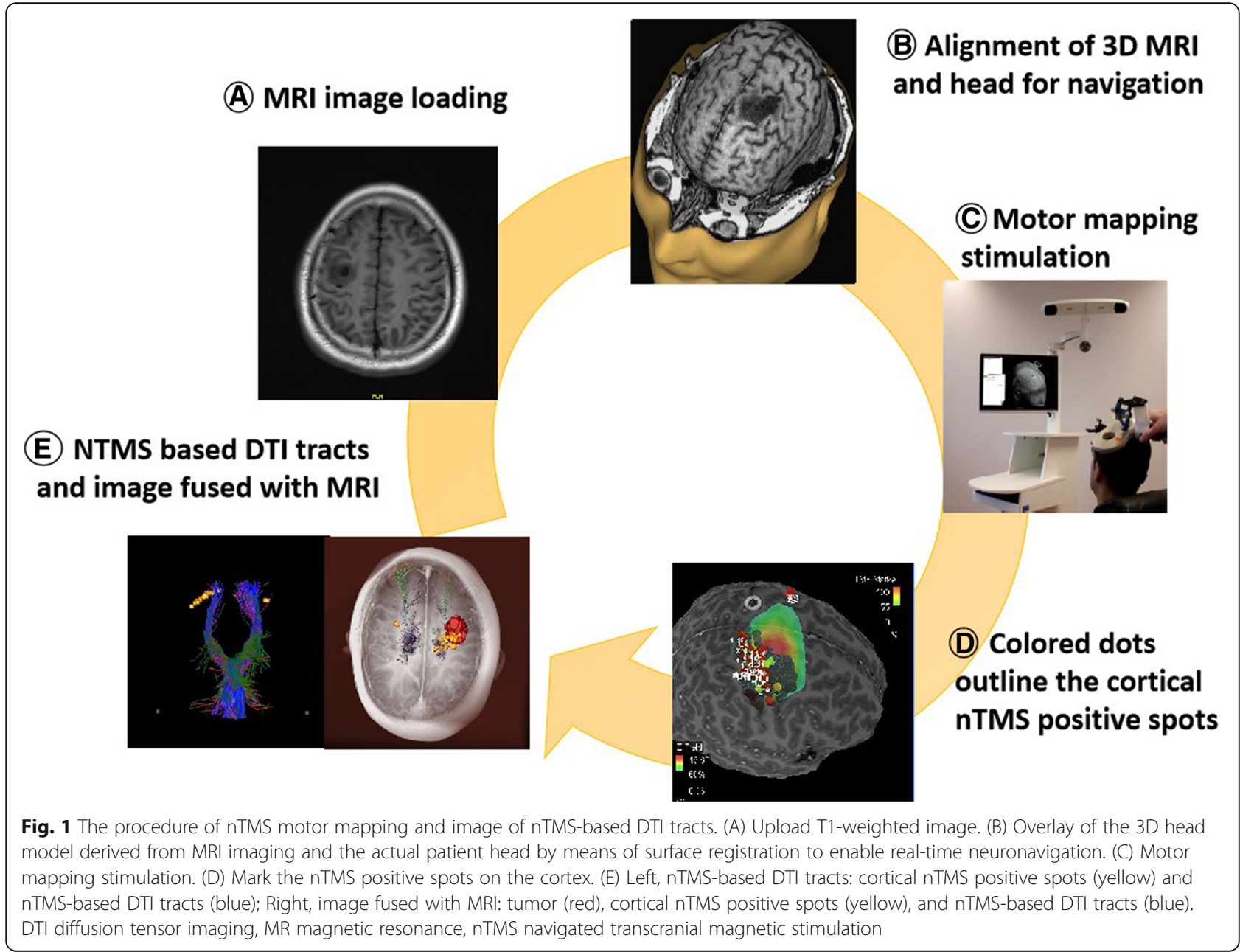

before operation, which is of great significance to the formulation of surgical plans. Preoperative localization of language function using nTMS is helpful to reduce the incision and improve the accuracy of intraoperative cortical electrical stimulation; therefore, functioning language regions under intraoperative examination should be expanded and not just limited to known anatomic areas [42]. Language mapping by nrTMS can help identify language-eloquent areas for patients not eligible for awake surgery and can be used for smaller craniotomies and focused intraoperative DES, increasing efficiency and safety [31].

NTMS mapping is highly reliable and can significantly shorten intraoperative DES mapping, but it still can not replace DES or interoperative monitoring (IOM) completely. On the contrary, nTMS serves as a valuable supplement for IOM but not a competing modality [43]. They should be used together to optimize the patient's selection and approach planning, accordingly accelerating the surgery and having better oncological and functional outcomes $[15,44]$.

\section{nTMS and fMRI}

At present, fMRI is the most frequently applied imaging methods for motor and linguistic function localization before operation. Compared with fMRI, nTMS imaging has better time resolution and does not require complex post-processing analysis. It also can be applied to patients with claustrophobia who can not complete the MRI examination [45]. The advantages and disadvantages of nTMS compared to fMRI and DES are shown in Table 1.

Researches showed that fMRI was less reliable for motor mapping in patients with brain tumor, especially in the regions near the lesion [46, 47]. NTMS showed a better tracking efficiency than fMRI in cases that the cortical tract origin was very close to a brain tumor probably because the tumor influenced the neurovascular coupling. When blood oxygenated level-dependent signal physiology was altered, nTMS appeared to be a better option [48].

Scientists have reported the combined use of nTMS and diffusion tensor imaging fiber tracking (DTI-FT) in the cortical spinal tract (CST) [48]. The DTI-FT based on nTMS is proved to be a more dependable and precise 
Table 1 Comparison among nTMS, DES, and fMRI

\begin{tabular}{|c|c|c|c|}
\hline & nTMS & DES & fMRI \\
\hline Safety issue & Safe, noninvasive & Invasive & Safe, noninvasive \\
\hline Accuracy & High accuracy & $\begin{array}{l}\text { Gold standard for mapping of } \\
\text { motor and language }\end{array}$ & $\begin{array}{l}\text { Accuracy is affected by patient's cooperation } \\
\text { Less reliable for motor mapping in patients } \\
\text { with brain tumor }\end{array}$ \\
\hline Maneuverability & $\begin{array}{l}\text { Simple } \\
\text { Do not need patient's cooperation }\end{array}$ & Complex & $\begin{array}{l}\text { Require complex post-processing analysis } \\
\text { Need patient's cooperation }\end{array}$ \\
\hline Expenses & Low & High & Low \\
\hline Level of comfort & $\begin{array}{l}\text { Well tolerated in motor mapping } \\
\text { A little uncomfortable in language mapping }\end{array}$ & Invasive & $\begin{array}{l}\text { Comfortable but can not be applied to } \\
\text { claustrophobic patients }\end{array}$ \\
\hline
\end{tabular}

tool to reconstruct the CST compared with the standard anatomical tractography. The functional and anatomical data acquired from the somatotopic reconstruction make it possible to assess the spatial correlation between the motor fibers and the lesion and improve the evaluation of the risks of tumor resection. In addition, the nTMS-based DTI-FT of the CST is able to use during surgery as a guide for orienting DES and lesion resection. Recent study showed that nTMS mapping especially nTMS-based DTI-FT can be served as a suitable surgical technique for motor-eloquent lesions and is likely to promote the risk/benefit analysis, resection extent, and outcomes [48-50].

The identified language-positive nrTMS spots can also be used as regions of interest (ROIs) for nTMS-based DTI-FT. Sollmann et al. did preoperative nrTMS language mapping in 40 patients and defined each error categories separately as a ROI, which was used for function-specific nTMS-based DTI-FT. Their results suggested that using different error categories as ROIs could result in better guidance during operation [51].

\section{Clinical use of nTMS in neurosurgery}

It is a great challenge for neurosurgeons and patients to perform a brain tumor resection located at or near the motor eloquent area. In brain tumor surgery, preserving neurological function and taking maximal resection are major principles, and neurosurgeons frequently encounter a dilemma between conservation of motor function and completeness of resection. Therefore, in order to reduce motor deficits, preoperative functional localization of nTMS is necessary. Intracranial lesions, such as ischemia or tumors, are likely to result in the displacement and remodeling of motor and speech areas. NTMS can accurately determine the displaced functional areas, thus providing assistance for surgical planning [52]. It is reported that nTMS not just affects indication and planning in surgery but also results in a higher rate of gross total resection and a lower rate of surgery-related paresis. Expanding indications for surgery and the extent of resection according to nTMS results enables more patients to undergo surgery and lead to better outcomes and high survival rates [44].

Krieg compared the surgical outcome of patients with motor eloquent metastatic lesions who received preoperative nTMS-based motor mapping with those that did not. Patients receiving nTMS had a lower rate of residual tumor, smaller craniotomies, shorter operation time, and decreased surgery-related paresis [53]. Krieg comprehensively studied the impact of preoperative motor mapping by nTMS on different clinical outcome parameters within a homogeneous cohort of high-grade glioma patients. The results demonstrated that patients who underwent nTMS preoperative mapping had smaller craniotomies and less residual tumor tissues [54]. Picht used nTMS in a group of patients with gliomas and found nTMS mapping could change the therapy planning into early and more extensive resection. The median change of tumor volume from baseline to 1 year was $-83 \%$ in the nTMS group, but $+12 \%$ in the comparison group [55].

Functional mapping with nTMS is available not only in tumorous brain lesions, but also within hypervascularized cortical areas [56]. Many studies had shown that nTMS can serve as a powerful tool to schedule planning prior to surgery for patients with arteriovenous malformation and cavernous angiomas, and it can also help optimize treatment planning [36, 57].

The mapping based on nTMS can visualize the language network more efficiently and can also detect cortical plasticity induced by an intra-hemispheric tumor-induced cortical plasticity. It can be used to formulate a tailored surgical plan which could preserve language function after the surgery. This tool could play a supplementary role for neurosurgeons in dealing with patients with potential language-eloquent tumors, but not applicable for awake surgery [58].

Besides surgical planning, the nTMS data and nTMS-based tractography are far beyond its current application. Schwendner implemented nTMS motor mapping in patients with intracranial metastases during routine radiotherapy. The results showed that it can significantly reduce the dose applied to the motor cortex 
while not affecting the treatment doses for the planning target volume [59].

NTMS can also be used in the prediction of recovery of paralysis. One study demonstrates that nTMS results before and after surgery have the potential to predict the motor function recovery after glioma resection. At 1 week after surgery, positive MEPs induced by nTMS signify a better recovery from postsurgical neurological deficits [60]. Peters examined the association of MEPs with lower extremities outcomes in a well-defined cohort of chronic, stable stroke survivors. They found MEP latency appears to be an indicator of lower extremities impairment and gait [61].

\section{Broad application of nTMS}

Single-pulse TMS can provide insight into the excitability and integrity of the CST [62], and paired-pulse TMS can provide insight into the excitability and integrity of corticocortical connections [63]. Common evaluation parameters of MEP include amplitude, latency, and central motor conduction time [64]. In clinical application, location and strength of the electric field could result in alterations in MEP amplitudes. Schmidt et al. demonstrated that the variability of cortical spinal excitability (CSE) is decreased by controlling the physical parameters via navigation. NTMS can help accurately identify and sustain nTMS coil's location to the targeted muscle's representation on the cortex, and this allows increasing the accuracy of nTMS motor mapping and the assessing of CSE [65].

Studies have used nTMS to evaluate the function of upper motor neurons in patients with amyotrophic lateral sclerosis (ALS). The RMT was significantly higher in ALS patients, and the mean motor mapping areas were smaller in patients with ALS than in controls. They found nTMS is a promising method for assessing upper motor neuron function in ALS and may clarify the pathogenic process of neurodegeneration and help establish novel diagnostic and prognostic models [66].

In recent years, preservation of language and motor function had been prioritized over other necessary functions. Several centers also used TMS to map further essential brain functions [5, 67]. Since TMS has been applied for mapping of neuropsychological cortical function, it seems feasible to map different neuropsychological functions more accurately by nTMS. It is reported that nTMS is available to accurately map calculation function at cortex [68] and cortical face processing function in healthy subjects [69].

An international investigation obtained level A evidence in determining the efficacy of repetitive TMS (rTMS) on neuropathic pain $[70,71]$. In most rTMS studies, stimulating the motor cortex at the opposite side of the aching side showed analgesic effects, regardless of the location of the pain [72, 73]. Few studies reported using image-guided navigated systems to identify motor cortex in pain treatment by rTMS [71, 74]. An image-guided navigated procedure might provide a customized solution for each patient receiving rTMS pain treatment. In one study, the analgesic effects produced by nrTMS were compared with those by non-navigated rTMS, and they found that there was a prolonged effect after stimulating the painful limb's cortical motor representation by nrTMS [71].

\section{Limitation of nTMS}

It should be noted that nTMS can produce mapping of cortical areas that are close to the surface and therefore reachable by the magnetic field. This signifies that mapping frontobasal and temporomesial gyri are not available, and similar to that, the mapping of the brain region covered by big meningiomas or big arachnoid cysts is unavailable.

\section{Conclusions}

NTMS is the latest technology in the functional localization of the cerebral cortex, which plays an important role in surgical planning, patient consultation, and risk assessment. The results show that the accuracy of nTMS in locating motor and language functions is similar to that of DES, higher than that of fMRI, and it is easy to operate. Therefore, it is recommended that nTMS can be used routinely to locate functional areas in patients with occupied functional areas. NTMS is a useful supplement for established IOM workflows while planning and conducting surgery in suspected motor eloquent regions. NTMS has high application value in the treatment of neurological and psychiatric diseases.

\section{Abbreviations}

3D: Three dimension; ALS: Amyotrophic lateral sclerosis; CSE: Cortical spinal excitability; CST: Cortical spinal tract; DES: Direct electrical cortical stimulation; DTIFT: Diffusion tensor imaging fiber tracking; EMG: Electromyogram; En-TMS: Electricfield navigated transcranial magnetic stimulation; fMRl: Function magnetic resonance imaging; IOM: Interoperative monitoring; Ln-TMS: Line-navigated transcranial magnetic stimulation; MEP: Motor-evoked potential; MRI: Magnetic resonance imaging; nTMS: Navigated transcranial magnetic stimulation; RMT: Resting motor threshold; ROl: Regions of interest; rTMS: Repetitive transcranial magnetic stimulation; TMS: Transcranial magnetic stimulation

\section{Acknowledgements}

Not applicable

Funding

Not applicable

\section{Availability of data and materials}

Not applicable

\section{Authors' contributions}

FXJ, LXZ, and ZYL contributed to the conception and design of the study. $F X J, L M G$, and $L C Y$ contributed to the acquisition and analysis of the data. FXJ and LXZ contributed to the drafting of the manuscript. FXJ, LXZ, and $Z Y L$ contributed to the revising of the manuscript critically for important intellectual content. FXJ, LMG, LCY, and ZYL contributed to the approval of the version of the manuscript to be published. All authors read and approved the final manuscript.

Ethics approval and consent to participate Not applicable 


\section{Consent for publication}

Not applicable

\section{Competing interests}

The authors declare that they have no competing interests.

\section{Author details}

'Department of Neurology, Peking University International Hospital, 1 Life Science St, Changping District, Beijing 102206, China. ${ }^{2}$ Neurosurgery Center Beijing Tiantan Hospital, Capital Medical University, Beijing 100070, China. ${ }^{3}$ Department of Neurosurgery, Peking University International Hospital, Beijing 102206, China. ${ }^{4}$ Department of Neurology, Peking University People's Hospital, Beijing 100044, China.

\section{Received: 14 January 2019 Accepted: 25 April 2019}

\section{Published online: 21 May 2019}

\section{References}

1. Barker AT, Jalinous R, Freeston IL. Non-invasive magnetic stimulation of human motor cortex. Lancet. 1985;325(8437):1106-7.

2. Rossini PM, Rossi S. Transcranial magnetic stimulation: diagnostic, therapeutic, and research potential. Neurology. 2007;68(7):484-8.

3. Pascual LA, Gates JR, Dhuna A. Induction of speech arrest and counting errors with rapid-rate transcranial magnetic stimulation. Neurology. 1991; 41(5):697-702

4. Seyal M, Siddigui I, Hundal NS. Suppression of spatial localization of a cutaneous stimulus following transcranial magnetic pulse stimulation of the sensorimotor cortex. Electroencephalogr Clin Neurophysiol. 1997;105:24-8.

5. Geogrge MS, Wassermann EM, Williams WA, et al. Changes in mood and hormone levels after rapid-rate transcranial magnetic stimulation (rTMS) of the prefrontal cortex. J Neuropsychiatry Clin Neurosci. 1996;8(2):172-80.

6. Grafman J, Wassermann E. Transcranial magnetic stimulation can measure and modulate learning and memory. Neuropsychologia. 1998;37(2):159-67.

7. Ahdab $R$, Ayache SS, Brugières $P$, et al. The hand motor hotspot is not always located in the hand knob: a neuronavigated transcranial magnetic stimulation study. Brain Topogr. 2016:29(4):590-7.

8. Neggers SF, Langerak TR, Schutter DJ, et al. A stereotactic method for imageguided transcranial magnetic stimulation validated with fMRI and motor-evoked potentials. Neuroimage. 2004;21:1805-17.

9. Paus T. Imaging the brain before, during, and after transcranial magnetic stimulation. Neuropsychologia. 1998;37(2):219-24.

10. Coburger J, Musahl C, Henkes $\mathrm{H}$, et al. Comparison of navigated transcranial magnetic stimulation and functional magnetic resonance imaging for preoperative mapping in rolandic tumor surgery. Neurosurg Rev. 2013;36(1):65-75.

11. Picht T, Schmidt S, Brandt $\mathrm{S}$, et al. Preoperative functional mapping for rolandic brain tumor surgery: comparison of navigated transcranial magnetic stimulation to direct cortical stimulation. Neurosurgery. 2011;69(3): $581-8$.

12. Forster MT, Hattingen E, Senft C, Gasser T, et al. Navigated transcranial magnetic stimulation and functional magnetic resonance imaging: advanced adjuncts in preoperative planning for central region tumors. Neurosurgery. 2011;68:1317-24

13. Tarapore PE, Tate MC, Findlay AM, et al. Preoperative multimodal motor mapping: a comparison of magnetoencephalography imaging, navigated transcranial magnetic stimulation, and direct cortical stimulation [J]. J Neurosurg. 2012;117(2):354-62.

14. Krieg SM, Shiban E, Buchmann N, et al. Utility of presurgical navigated transcranial magnetic brain stimulation for the resection of tumors in eloquent motor areas. J Neurosurg. 2012;116(5):994.

15. Krieg SM, Sollmann N, Obermueller T, et al. Changing the clinical course of glioma patients by preoperative motor mapping with navigated transcranial magnetic brain stimulation. BMC Cancer. 2015;15(1):231.

16. Raffa G, Bährend I, Schneider H, et al. A novel technique for region and linguistic specific nTMS-based DTI fiber tracking of language pathways in brain tumor patients [J]. Front Neurosci. 2016:10:552.

17. Ruohonen J, Karhu J. Navigated transcranial magnetic stimulation. Neurophysiol Clin. 2010:40:7-17.

18. Sollmann N, Goblirsch-Kolb MF, Ille S, et al. Comparison between electricfeld-navigated and line-navigated TMS for cortical motor mapping in patients with brain tumors. Acta Neurochir. 2016;158:2277-89.
19. Picht T. Current and potential utility of transcranial magnetic stimulation in the diagnostics before brain tumor surgery. CNS Oncol. 2014;3:299-310.

20. Mäkelä, Teemu, Vitikainen AM, Laakso A, et al. Integrating nTMS Data into a Radiology Picture Archiving System. J Digit Imaging. 2015;28(4):428-32.

21. Krieg SM, Shiban E, Buchmann N, et al. Presurgical navigated transcranial magnetic brain stimulation for recurrent gliomas in motor eloquent areas. Clin Neurophysiol. 2013;124:522-7.

22. Picht $T$, Frey $D$, Thieme $S$, et al. Presurgical navigated TMS motor cortex mapping improves outcome in glioblastoma surgery: a controlled observational study. J Neuro-Oncol. 2016;126:535-43.

23. Mylius $V$, Ayache SS, Ahdab R, et al. Definition of DLPFC and M1 according to anatomical landmarks for navigated brain stimulation: inter-rater reliability, accuracy, and influence of gender and age. Neurolmage. 2013;78:224-32.

24. Ahdab R, Ayache SS, Farhat WH, et al. Reappraisal of the anatomical landmarks of motor and premotor cortical regions for image-guided brain navigation in TMS practice. Hum Brain Mapp. 2014;35(5):S200

25. Rossini PM, Barker AT, Berardelli A, et al. Non-invasive electrical and magnetic stimulation of the brain, spinal cord and roots: basic principles and procedures for routine clinical application. Report of an IFCN committee. Electroencephalogr Clin Neurophysiol. 1994;91(2):79-92.

26. Krieg SM, Lioumis P, Makela JP, et al. Protocol for motor and language mapping by navigated TMS in patients and healthy volunteers; workshop report. Acta Neurochir. 2017:159:1187-95.

27. Lioumis $\mathrm{P}, \mathrm{Zhdanov} \mathrm{A}$, Mäkelä N, et al. A novel approach for documenting naming errors induced by navigated transcranial magnetic stimulation. J Neurosci Methods. 2012;204:349-54.

28. Hernandez-Pavon JC, Mäkelä N, Lehtinen $\mathrm{H}$, et al. Effects of navigated TMS on object and action naming. Front Hum Neurosci. 2014;8:660.

29. Corina DP, Loudermilk BC, Detwiler $L$, et al. Analysis of naming errors during cortical stimulation mapping: implications for models of language representation. Brain Lang. 2010;115:101-12.

30. Picht T, Krieg SM, Sollmann N, et al. A comparison of language mapping by preoperative navigated transcranial magnetic stimulation and direct cortical stimulation during awake surgery. Neurosurgery. 2013;72(5):808-19.

31. Rossi S, Hallett M, Rossini PM, et al. Safety, ethical considerations, and application guidelines for the use of transcranial magnetic stimulation in clinical practice and research [J]. Clin Neurophysiol. 2009;120(12): 2008-39.

32. Tarapore PE, Picht T, Bulubas L, et al. Safety and tolerability of navigated TMS for preoperative mapping in neurosurgical patient. Clin Neurophysiol. 2016;127(3):1895-900.

33. Tarapore PE, Picht T, Bulubas L, et al. Safety and tolerability of navigated TMS in healthy volunteers [J]. Clin Neurophysiol. 2016;127(3): 1916-8.

34. Schrader LM, Stern JM, Koski L, et al. Seizure incidence during single-and paired-pulse transcranial magnetic stimulation (TMS) in individuals with epilepsy. Clin Neurophysiol. 2004;115(12):2728-37.

35. De Witt Hamer PC, Robles SG, Zwinderman AH, et al. Impact of intraoperative stimulation brain mapping on glioma surgery outcome: a meta-analysis. J Clin Oncol. 2012;30:2559-65.

36. Paiva WS, Fonoff ET, Marcolin MA, et al. Navigated transcranial magnetic stimulation in preoperative planning for the treatment of motor area cavernous angiomas. Neuropsychiatr Dis Treat. 2013:9:1885.

37. Takahashi S, Vajkoczy P, Picht T. Navigated transcranial magnetic stimulation for mapping the motor cortex in patients with rolandic brain tumors. Neurosurg Focus. 2013;34(4):E3.

38. Paiva WS, Fonoff ET, Marcolin MA, et al. Cortical mapping with navigated transcranial magnetic stimulation in low-grade glioma surgery. Neuropsychiatr Dis Treat. 2012;8:197-201.

39. Ille S, Kulchytska N, Sollmann N, et al. Hemispheric language dominance measured by repetitive navigated transcranial magnetic stimulation and postoperative course of language function in brain tumor patients. Neuropsychologia. 2016;91:50-60

40. Krieg SM, Sollmann N, Tanigawa N, et al. Cortical distribution of speech and language errors investigated by visual object naming and navigated transcranial magnetic stimulation. Brain Struct Funct. 2016;221(4):2259-86.

41. Tarapore PE, Findlay AM, Honma SM, et al. Language mapping with navigated repetitive TMS: proof of technique and validation. Neuroimage. 2013;82:260-72

42. Pulvermüller $F$, Fadiga L. Active perception: sensorimotor circuits as a cortical basis for language. Nat Rev Neurosci. 2010;11(5):351. 
43. Sollmann N, Negwer C, Tussis L, et al. Interhemispheric connectivity revealed by diffusion tensor imaging fiber tracking derived from navigated transcranial magnetic stimulation maps as a sign of language function at risk in patients with brain tumors [J]. J Neurosurg. 2017;126(1):222-33.

44. Frey $D$, Schilt $S$, Strack $V$, et al. Navigated transcranial magnetic stimulation improves the treatment outcome in patients with brain tumors in motor eloquent locations. Neuro-Oncology. 2014;16(10):1365-72.

45. Ottenhausen M, Krieg SM, Meyer B, et al. Functional preoperative and intraoperative mapping and monitoring: increasing safety and efficacy in glioma surgery. Neurosurg Focus. 2015;38(1):E3.

46. Giussani C, Roux FE, Ojemann J, et al. Is preoperative functional magnetic resonance imaging reliable for language areas mapping in brain tumor surgery? Review of language functional magnetic resonance imaging and direct cortical stimulation correlation studies. Neurosurgery. 2010;66(1):113-20.

47. Ille S, Sollmann N, Hauck T, et al. Impairment of preoperative language mapping by lesion location: a functional magnetic resonance imaging, navigated transcranial magnetic stimulation, and direct cortical stimulation study. J Neurosurg. 2015;123(2):314-24.

48. Buchmann N, Gempt J, Stoffel M, et al. Utility of diffusion tensor-imaged (DTI) motor fiber tracking for the resection of intracranial tumors near the corticospinal tract. Acta Neurochir. 2011;153(1):68-74.

49. Lucas CW, Tursunova I, Neuschmelting V, et al. Functional MRI vs. navigated TMS to optimize M1 seed volume delineation for DTI tractography. A prospective study in patients with brain tumours adjacent to the corticospinal tract. Neurolmage: Clinical. 2017;13:297-309.

50. Raffa G, Conti A, Scibilia A, et al. Functional reconstruction of motor and language pathways based on navigated transcranial magnetic stimulation and DTI fiber tracking for the preoperative planning of low grade glioma surgery: a new tool for preservation and restoration of eloquent networks. Acta Neurochir Suppl. 2017:251-61.

51. Sollmann N, Zhang H, Schramm S, et al. Function-specific Tractography of language pathways based on nTMS mapping in patients with Supratentorial lesions. Clin Neuroradiol. 2018:1-13.

52. Bulubas L, Sollmann N, Tanigawa N, et al. Reorganization of motor representations in patients with brain lesions: a navigated transcranial magnetic stimulation study. Brain Topogr. 2018;31(2):288-99.

53. Krieg SM, Picht T, Sollmann N, et al. Resection of motor eloquent metastases aided by preoperative nTMs-based motor maps-comparison of two observational cohorts [J]. Front Oncol. 2016;6:261.

54. Krieg SM, Sabih J, Bulubasova L, et al. Preoperative motor mapping by navigated transcranial magnetic brain stimulation improves outcome for motor eloquent lesions. Neuro-oncology. 2014;16(9):1274.

55. Picht T, Schulz J, Vajkoczy P. The preoperative use of navigated transcranial magnetic stimulation facilitates early resection of suspected low-grade gliomas in the motor cortex. Acta Neurochir. 2013;155(10):1813-21.

56. Kato N, Schilt S, Schneider H, et al. Functional brain mapping of patients with arteriovenous malformations using navigated transcranial magnetic stimulation: first experience in ten patients [J]. Acta Neurochir. 2014;156(5):885-95.

57. Ille S, Picht T, Shiban E, et al. The impact of nTMS mapping on treatment of brain AVMs. Acta Neurochir. 2018;160(3):567-78.

58. Raffa G, Quattropani MC, Scibilia A, et al. Surgery of language-eloquent tumors in patients not eligible for awake surgery: the impact of a protocol based on navigated transcranial magnetic stimulation on presurgical planning and language outcome, with evidence of tumor-induced intrahemispheric plasticity. Clin Neurol Neurosurg. 2018;168:127-39.

59. Schwendner MJ, Sollmann N, Diehl CD, et al. The role of navigated transcranial magnetic stimulation motor mapping in adjuvant radiotherapy planning in patients with supratentorial brain metastases. Front Oncol. 2018;8.

60. Takakura T, Muragaki $Y$, Tamura M, et al. Navigated transcranial magnetic stimulation for glioma removal: prognostic value in motor function recovery from postsurgical neurological deficits [J]. J Neurosurg. 2017;127(4):877-91.

61. Peters HT, Kari D, Samir B, et al. Navigated transcranial magnetic stimulation: a biologically based assay of lower extremity impairment and gait velocity. Neural Plasticity. 2017;2017:1-7.

62. Kobayashi M, Pascual-Leone A. Transcranial magnetic stimulation in neurology [J]. Lancet Neurol. 2003;2(3):145-56.

63. Ziemann ULF, Rothwell JC, Ridding MC. Interaction between intracortical inhibition and facilitation in human motor cortex. J Physiol. 1996:496(3):873-81.

64. Groppa S, Schlaak BH, Münchau A, et al. The human dorsal premotor cortex facilitates the excitability of ipsilateral primary motor cortex via a short latency cortico-cortical route. Hum Brain Mapp. 2012;33(2):419-30.
65. Schmidt S, Bathe-Peters R, Fleischmann R, et al. Nonphysiological factors in navigated TMS studies; confounding covariates and valid intracortical estimates. Hum Brain Mapp. 2015;36(1):40-9.

66. Chervyakov AV, Bakulin IS, Savitskaya NG, et al. Navigated transcranial magnetic stimulation in amyotrophic lateral sclerosis. Muscle Nerve. 2015; 51(1):125-31.

67. Hallett M, Di lorio R, Rossini PM, et al. Contribution of transcranial magnetic stimulation to assessment of brain connectivity and networks. Clin Neurophysiol. 2017;128(11):2125-39.

68. Maurer S, Tanigawa N, Sollmann N, et al. Non-invasive mapping of calculation function by repetitive navigated transcranial magnetic stimulation. Brain Struct Funct. 2016;221(8):3927-47.

69. Maurer S, Giglhuber K, Sollmann N, et al. Non-invasive mapping of face processing by navigated transcranial magnetic stimulation. Front Hum Neurosci. 2017:11:4.

70. Lefaucheur JP. The use of repetitive transcranial magnetic stimulation (rTMS) in chronic neuropathic pain. Neurophysiol Clin. 2006:36:117-24.

71. Ayache SS, Ahdab R, Chalah MA, et al. Analgesic effects of navigated motor cortex rTMS in patients with chronic neuropathic pain. Eur J Pain. 2016;20: $1413-22$.

72. André-Obadia N, Magnin M, Garcia-Larrea L. On the importance of placebo timing in rTMS studies for pain relief. Pain. 2011;152:1233-7.

73. Kang BS, Shin HI, Bang MS. Effect of repetitive transcranial magnetic stimulation over the hand motor cortical area on central pain after spinal cord injury. Arch Phys Med Rehabil. 2009;90:1766-71.

74. Hodaj H, Alibeu JP, Payen JF, et al. Treatment of chronic facial pain including cluster headache by repetitive transcranial magnetic stimulation of the motor cortex with maintenance sessions: a naturalistic study. Brain Stimul. 2015;8(4):801-7.

\section{Ready to submit your research? Choose BMC and benefit from:}

- fast, convenient online submission

- thorough peer review by experienced researchers in your field

- rapid publication on acceptance

- support for research data, including large and complex data types

- gold Open Access which fosters wider collaboration and increased citations

- maximum visibility for your research: over $100 \mathrm{M}$ website views per year

At BMC, research is always in progress.

Learn more biomedcentral.com/submission 